\title{
Stent patency and outcome of TIPS through the left versus the right portal branches
}

\author{
Mohamed S. Alwarraky ${ }^{1,2^{*}}$, Hasan A. Elzohary ${ }^{3}$, Mohamed A. Melegy ${ }^{3}$ and Anwar Mohamed $^{3}$
}

\begin{abstract}
Background: Our purpose is to compare the stent patency and clinical outcome of trans-jugular intra-hepatic porto-systemic shunt (TIPS) through the left branch portal vein (TIPS-LPV) to the standard TIPS through the right branch (TIPS-RPV). We retrospectively reviewed all patients $(n=54)$ with refractory portal hypertension who were subjected to TIPS-LPV at our institute (TIPS-LPV) between 2016 and 2018. These patients were matched with 56 control patients treated with the standard TIPS-RPV (TIPS-RPV). The 2 groups were compared regarding the stent patency rate, encephalopathy, and re-interventions for 1 year after the procedure.

Results: TIPS-LPV group showed 12 months higher patency rate $(90.7 \%$ compared to $73.2 \%)(P<0.005)$. The number of the encephalopathy attacks in the TIPS-LPV group was significantly lower than that of the TIPS-RPV group at 6 and 12 months of follow-up $[P=0.012$ and 0.036 , respectively]. Re-bleeding and improvement of ascites were the same in the two groups $[P>0.05]$. Patients underwent TIPS-LPV needed less re-interventions and required less hospitalizations than those with TIPS-RPV $[P=0.039$ and $P=0.03$, respectively].
\end{abstract}

Conclusion: The new TIPS approach is to extend the stent to LPV. This new TIPS-LPV approach showed the same clinical efficiency as the standard TIPS-RPV in treating variceal bleeding and ascites. However, it proved a better stent patency with lower rates of re-interventions, encephalopathy, and hospital admissions than TIPS through the right branch.

Keywords: Stent patency, TIPS, Left portal vein, Encephalopathy, Re-intervention

\section{Background}

TIPS still has the advantage of being less invasive for shunt implantation, yet, with similar decompressive effects on of the portal circulation as the surgical shunts do $[1,2]$. Randomized controlled trials and metaanalyses have shown that the TIPS is superior to endoscopic therapy in refractory bleeding, preventing variceal re-bleeding and difficult cases of recurrent or refractory ascites [3, 4]. However, many authors reported frequent

\footnotetext{
* Correspondence: drwarraky@yahoo.com

${ }^{1}$ Radiology Department, National Liver Institute, Menoufia University,

Menoufia, Shebin Al-Kom, Egypt

2Department of Diagnostic \& Interventional Imaging, National Liver Institute, Menoufia University, Yassin Abdelghaffar St., Menoufia, Shebin Al-Kom, Egypt Full list of author information is available at the end of the article
}

TIPS dysfunctions mandating repeated re-interventions during follow-up [5-7]. Also, there is an increased incidence of encephalopathy after TIPS $[8,9]$.

In the standard TIPS-RPV, the right portal is the only used approach and its cannulation is a critical step for the success of TIPS. Recently, the left approach has been tried in many centers. The authors reported that the outcome of the patient after TIPS is related to the technique used. They reported that the stent in the case of TIPS-RPV is usually more liable to inevitable looping, angulation, and kinking causing frequent shunt dysfunction [10]. Moreover, stent decreases the perfusion of the larger portion of the liver (Rt. lobe segments represent $70 \%$ ). Thus, the hemodynamics of liver blood flow from the portal vein is affected to a greater extent when using 
the RPV approach [11, 12]. Stent dysfunction and effects on hemodynamics are the most important determining factors regarding TIPS efficiency in portal hypertension [13, 14]. On the other hand, cannulation of the left portal vein decreases the perfusion of the left lobe of the liver (supply 30\%) and may provide a straight course of the stent. Moreover, the quality and quantity in the portal vein help predict the patient's outcome after TIPS [15]. According to streamline flow theory, the splenic blood flow is usually directed to the left lobe while the intestinal blood supplies the right lobe. So, shunting from the left portal is associated with less incidence of hepatic coma [16].

Few studies have evaluated the efficacy of TIPS-LPV regarding the clinical outcome without detailed discussion of the patency of the stent in such a new technique $[17,18]$. Moreover, there is a lack of studies that compared to the TIPS-LPV to standard TIPS-RPV in this regard [19].

In this study, we aimed to compare the stent patency and clinical efficacy of TIPS with LPV shunt to those of RPV shunt.

\section{Methods}

\section{Study design and constitution of the groups}

This retrospective comparative study was conducted on patients who were subjected to TIPS from 2008 to 2018 in our hospital. Fifty-four consecutive patients with severe portal hypertension were subjected to TIPS to the LPV (TIPS-LPV group). Those patients were compared to 56 matching control group of patients selected from 1450 patients that were subjected to the standard TIPS to RPV in our institute (TIPSRPV group). The patients of the control group were selected anonymously and without knowledge of the results of our primary or secondary outcomes. Matching criteria between the two groups included age, gender, Child-Pugh score, etiology of cirrhosis as a cause of portal hypertension, symptoms of portal hypertension, and stent type. An informing consent was signed by all patients. Eligibility criteria in both groups were (1) diagnosis of liver cirrhosis clinically, radiologically, or by biopsy, (2) history of repeated upper GIT bleeding attacks or bleeding once of a massive amount $(1000 \mathrm{ml})$, (3) endoscopic confirmation that bleeding is of variceal source, and (4) age between 18 and 65 years. Exclusion criteria were (1) patients with large $\mathrm{HCC}$ or other malignancies in the liver, (2) portal vein thrombosis, (3) chronic renal failure, (4) severe systemic infection, (5) severe pulmonary hypertension, and (6) impaired cardiac contractility with ejection fraction less than $30 \%$.

\section{Technique of TIPS in both groups (to LPV and to RPV)}

The standard technique of placing a TIPS stent to establish a portosystemic shunt and the post-procedural management has been described previously [16, 17]. After mild intravenous sedation and analgesia (50 to $100 \mathrm{mg}$ of meperidine and 1 to $5 \mathrm{mg}$ of midazolam), a puncture needle was advanced transjugularly through the IVC.

In the TIPS-LPV group $(n=54)$, the metal TIPS needle (RUPS-100, Cook Inc., Bloomington, IN, USA) was pre-bent to $60^{\circ}$ and up $90^{\circ}$ in some cases. The RUPS needle was then turned $60^{\circ}$ anti-clockwise to be able to puncture the left branch of the portal vein from the left or middle hepatic vein. In the case of Budd-Chiari, we directly punctured the retro-hepatic cava to the liver parenchyma. Once the left portal was reached, the guidewire was advanced to reach the main portal vein. The balloon was advanced to dilate the tract to the left portal. The bare Wallstents (Medinvent, Schneider, Bülach, Switzerland) were implanted then dilated to 8 $\mathrm{mm}$ in diameter. We increased dilatation to $10 \mathrm{~mm}$ if the porto-caval pressure gradient was still high and the end-point of the gradient was little above or equal to 12 mmHg. The Wallstents were deployed with care not to reach the main trunk (Figs. 1, 2, and 3). In three cases, there were previous failures to recanalize an occluded right TIPS stents. In these cases, left parallel stents were inserted beside the occluded ones (Figs. 4 and 5).

In the standard TIPS-RPV group [ $n=56]$, the puncture was through the right or middle hepatic vein to the right branch of portal vein as routinely described. Similar metal cannula of RUPS-100 (Cook Inc., Bloomington, IN, USA) was used. The primary TIPS-needle curve was kept at $45^{\circ}$ displaying sufficient torque control. The bare Wallstents were implanted then dilated incrementally as in the case of TIPS-LPV (Fig. 6).

\section{Data collection}

All clinical, laboratory, and radiological data over 1 year were collected. TIPS occlusion, re-bleeding, reintervention, encephalopathy, and hospital re-admission were collected and tabulated.

When a stenosis or occlusion was suspected, the patient had to undergo revision with angiography and managed either by angioplasty, extra-stent insertion, or both. Comparison between the two groups took place regarding stent patency, re-intervention, encephalopathy, re-bleeding, and hospital re-admission.

\section{Study outcomes}

The primary outcome of our study was to compare the 6 and 12 months patency rates of TIPS-LPV and TIPSRPV as known by the routine Doppler studies. The secondary end-points were the clinical outcome regarding rebleeding and ascites. The encephalopathy, number of 


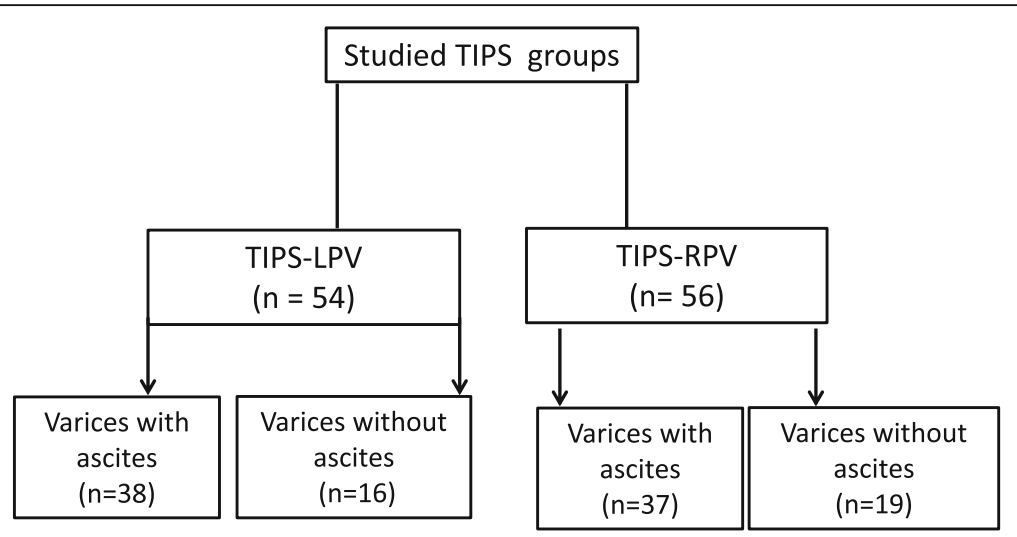

Fig. 1 Flowchart showing the patients in both TIPS groups

re-interventions, and hospital admissions were also determined and compared in both groups at 6 and 12 months.

\section{Statistical analysis}

Data analysis was conducted using SPSS v.24 software (SPSS Inc., Chicago, IL, USA). Continuous variables are presented as mean $\pm \mathrm{SD}$. Categorical variables are expressed as number (percentage). The normal distribution of the data was checked by the KolmogorovSmirnov test. If normally distributed, Student's $t$ test was used to compare the means of the outcomes. Comparisons of differences in the categorical data between groups were performed using the chi-square test. Continuous variables were analyzed by Student's $t$ test. $P$ value $<0.05$ was considered statistically significant in all these tests.

\section{Results}

\section{Patients' characteristics (Table 1)}

Of 54 patients of the TIPS-LPV group, 4 patients had Child-Pugh class A, 44 had class B, and 6 had class C.
The mean age was $47.4 \pm 6.2$ years, ranging from 38.0 to 58.8 years. For the 56 patients of the TIPS-RPV group, 5 patients had Child-Pugh class A, 46 had class B, and 5 had class $C$. The mean age was $46.4 \pm 5.8$ years, ranging from 36.0 to 62.8 years. The other demographic data are shown in (Table 1). There were no significant differences in the baseline characteristics between the two groups, including serum albumin, serum bilirubin, serum creatinine, prothrombin time, hemoglobin, and model of end-stage liver disease (MELD) scores.

\section{Stent patency rates in both groups and re-interventions} (Table 2)

By the end of 6 months, 51/54 patients showed patent stents in the TIPS-LPV group while $45 / 56$ patients in TIPS-RPV. So, the primary patency rate was significantly higher in the TIPS-LPV (94.4\%) compared to the patency rate of the TIPS-RPV $(80.3 \%)(P<0.01)$. Three patients out the in the TIPS-LPV showed stenosis/ occlusion while 11 patients in TIPS-RPV showed stenosis/occlusion, who were subjected to re-interventions for recanalization. The re-interventions in both groups

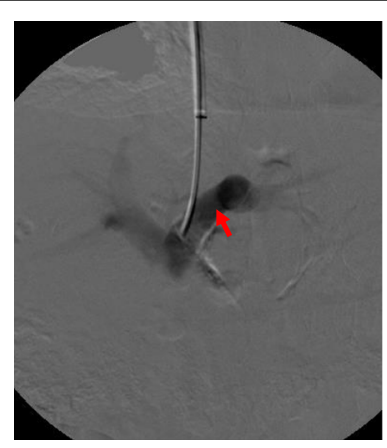

a

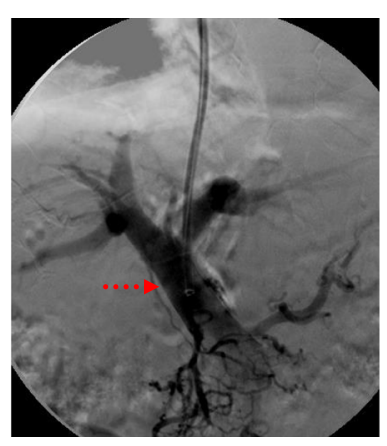

b

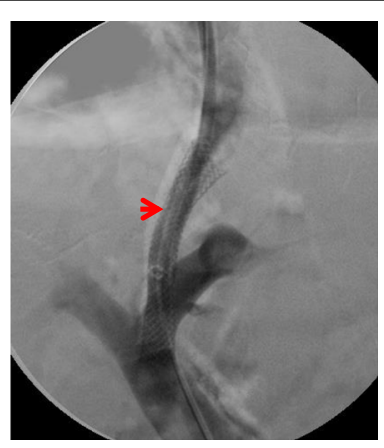

C

Fig. 2 TIPS case in the TIPS-LPV group. The sheath catheter containing the needle (arrow) was advanced inside the left portal (arrow) with limited left portography (a). Main portal (interrupted arrow) venograhy was done (b). The delivered Wallstent (arrowhead) was inserted with an apparent straight course and good flow shunting from left portal to cava (c) 


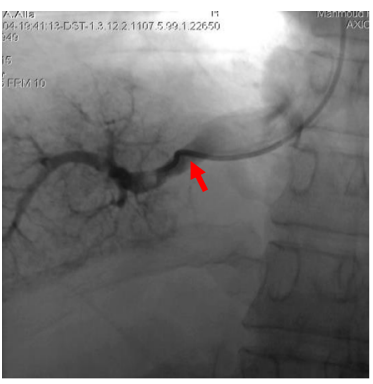

a

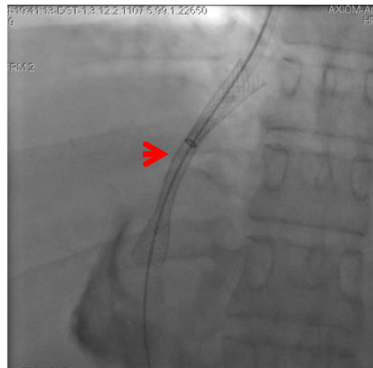

d

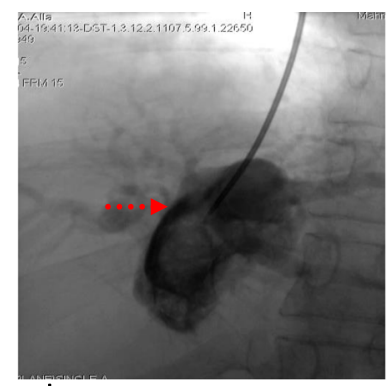

b

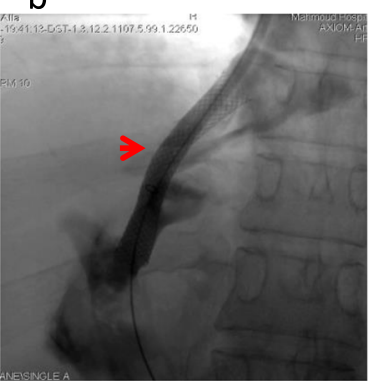

e

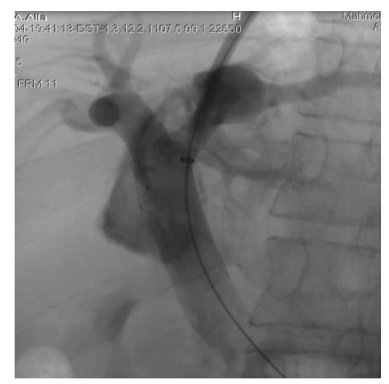

C

Fig. 3 Another TIPS case in the TIPS-LPV group. Middle hepatic (arrow) venography through a diagnostic catheter (a). The sheath catheter containing the needle was advanced inside the genu left portal (interrupted arrow) (b) with limited portography (a). Main portal venography was done with dilated portal vein (c). The delivered Wallstent (arrowhead) was inserted (d) with good flow shunting from left portal to cava (e)

consisted of balloon dilatation with or without instenting. Owing to the higher patency, TIPS-LPV showed a lower rate of 6-month occlusion/stenosis $(5.6 \%$ compared to $19.7 \% ; P<0.05)$ and required four reinterventions during follow-up compared to 13 interventions for the patients in TIPS-RPV $(P<0.05)$.

By the end of 12 months, 49/54 patients showed patent stents in the TIPS-LPV group while $41 / 56$ patients in TIPS-RPV. So, the primary patency rate was significantly higher in the TIPS-LPV (90.7\%) compared to the patency rate of the TIPS-RPV $(73.2 \%)(P<0.01)$. Three patients more in the TIPS-LPV group compared to 4 patients in TIPS-RPV showed stenosis/occlusion between the 6th and 12th months. Finally, the overall number of patients with stent dysfunction was 5 patients in TIPS-LPV compared to 15 patients in TIPS-RPV. Owing to the higher patency, TIPS-LPV showed a significantly lower rate of stenosis/occlusion $(9.3 \%$ compared to $26.8 \%(P<0.05)$. Regarding reinterventions, five patients in TIPS-LPV required five recanalizations during follow-up compared to 19 interventions for the 15 patients in TIPS-RPV (three patients required repeated interventions) $(P<0.05)$. In all patients, shunt patency or recanalization was restored with angioplasty and/or insertion of an extra-stent.

\section{Re-bleeding}

Re-bleeding showed no significant differences at any time points up to 1 year of follow-up. This result

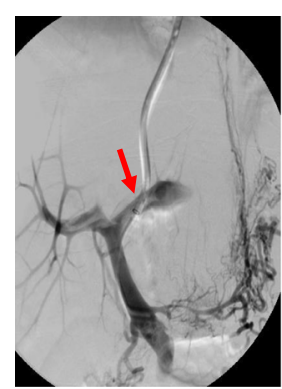

a

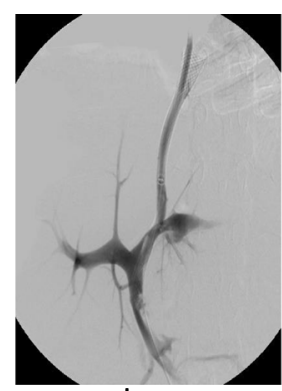

b

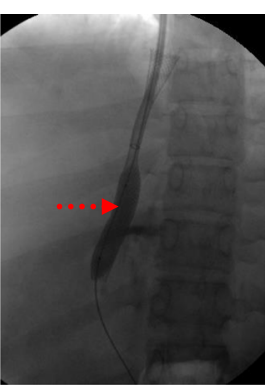

C

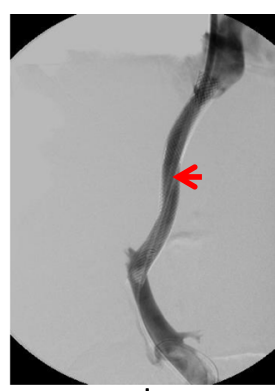

d

Fig. 4 A TIPS through the LPV in a male patient with ascites and varices. The puncture needle (arrow) inside the left portal branch (a). Subsequently, the catheter was introduced inside the main portal (b) with portography. Balloon (interrupted arrow) was introduced to dilate the stent (c) between the left portal and cava. The stent after dilatation (arrowhead) shows a straight course with an excellent flow (d) 


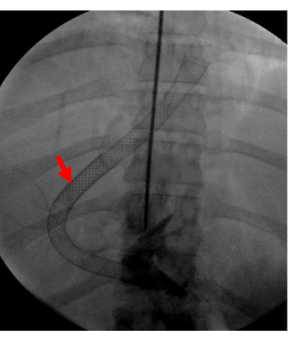

a

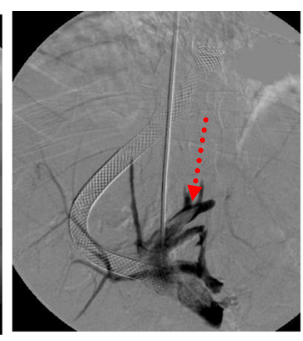

b

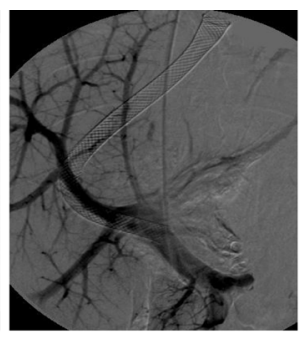

C

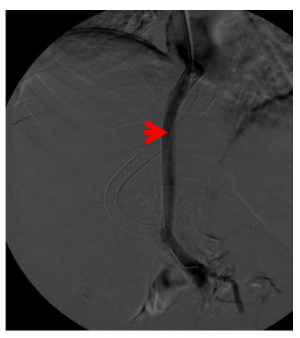

d

Fig. 5 Male patient with occluded long and curved (arrow) TIPS-shunt (a). Left portal vein was approached followed by left portography (interrupted arrow) (b). Then, the main portography was done to measure the pressure (c). Finally, the Wallstent (arrowhead) that was delivered shows a straight course between the left portal and cava with an excellent flow (d)

confirmed that both TIPS-LPV and TIPS-RPV can efficiently prevent variceal bleeding. In other words, there was no significant difference in re-bleeding incidences between the TIPS-LPV and the TIPS-RPV groups $(P=$ 0.493). During the follow-up of the TIPS-LPV group, 2 patients (2/54) experienced an episode of re-bleeding (one patient within 0-3 months, one within 6-12 months). In one patient, bleeding resulted of anticoagulant therapy while the other patient from variceal bleeding. The final numbers yielded a 12 -month rate of $4.7 \%$ for GIT bleeding after TIPS-LPV. In the TIPS-RPV group, three patients (3/56) experienced an episode of gastrointestinal bleeding (2 patients within 3-6 months and 1 within 12 months). Two cases were caused by varies and 1 by anticoagulant therapy, which makes the incidence of re-bleeding $5.7 \%$ after TIPS-RPV.

\section{Ascites}

The other indication of TIPS is to treat the ascites caused by portal hypertension. The patients included in this clinical study were not required to have ascites at the beginning of the study. In the TIPS-LPV group, there were 38 patients with different amounts of ascites, and in the TIPS-RPV group, there were 37 patients with different amounts of ascites before TIPS placement. Thus, there was no significant difference between the two TIPS groups $(P=0.316)$ (Table 1$)$. From this study, we also observed the effect of the two kinds of TIPS in alleviating ascites. Patients with pre-TIPS ascites routinely had the standard doses of diuretics post-TIPS for 1-2 weeks until ascites was significantly resolved. Lowdose diuretics such as spironolactone $(100 \mathrm{mg}$, once per day) and/or furosemide ( $20 \mathrm{mg}$, two times per day) were commonly administered in these cases. Paracentesis was unnecessary because the ascites was easily controlled by the standard doses of diuretics post-TIPS. There were $31 / 38(81.6 \%)$ patients in the TIPS-LPV group who had no detectable ascites by ultrasound within the 12 months of follow-up, including 19 patients within 0-3 months, 9 within 3-6 months, and 3 within $6-12$ months. However, in the TIPS-RPV group, there were $30 / 37$ (81\%) patients who had no ascites within 12 months of follow-up, including 22 patients within 0-3 months, 6 within 3-6 months, and 2 within 6-12 months. Both TIPS-LPV and TIPS-RPV exerted efficient effects on resolving ascites, and there was no significant difference in the treatment efficiency of ascites between the two groups $(P=0.167)$.

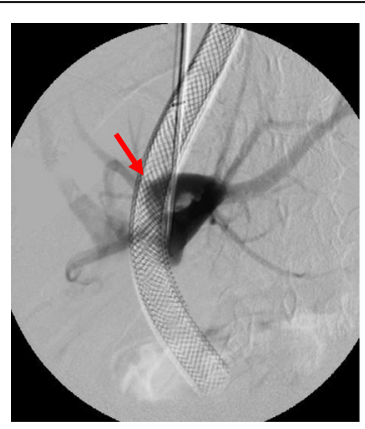

a

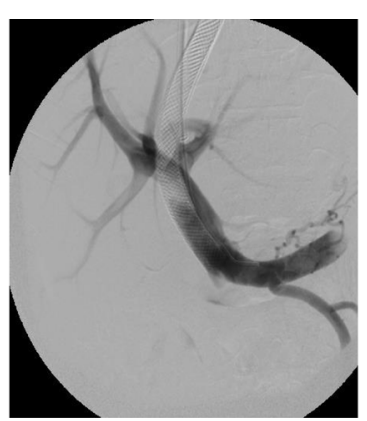

b

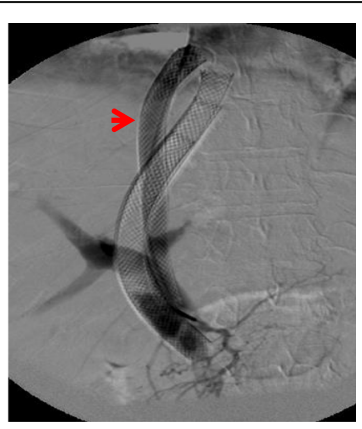

C

Fig. 6 Male patient with occluded standard TIPS-RPV (arrow). The left portal vein was approached followed by left portography (a). Then, the main portography was done to measure the pressure (b). Finally, the Wallstent (arrowhead) that was delivered shows a straight course between the left portal and cava with an excellent flow (c) 
Table 1 Demographic data of patients in both studied groups

\begin{tabular}{|c|c|c|}
\hline & TIPS-LPV & TIPS-RPV \\
\hline \multicolumn{3}{|l|}{ Clinical characteristics } \\
\hline Age (years) & $46.8 \pm 7.3$ & $43.7 \pm 8.2$ \\
\hline Range & $25-65$ & $22-65$ \\
\hline \multicolumn{3}{|l|}{ Gender $(n)$} \\
\hline Male & $38(70.3 \%)$ & $40(71.4 \%)$ \\
\hline Female & $16(29.6 \%)$ & $16(28.6 \%)$ \\
\hline \multicolumn{3}{|l|}{ Cirrhosis etiology $(n)$} \\
\hline Virus C & $28(51.9 \%)$ & $32(57.2 \%)$ \\
\hline Budd-Chiary & $22(40.7 \%)$ & $18(32.1 \%)$ \\
\hline Virus B & $4(7.4 \%)$ & $6(10.7 \%)$ \\
\hline \multicolumn{3}{|l|}{ Child (n) } \\
\hline A & $4(7.4 \%)$ & $5(7.6 \%)$ \\
\hline B & $44(81.4 \%)$ & $46(82.1 \%)$ \\
\hline C & $6(11.2 \%)$ & $5(11.3 \%)$ \\
\hline \multicolumn{3}{|l|}{ Indications of TIPS (n) } \\
\hline Bleeding with ascites & $38(71.4 \%)$ & $37(67.1 \%)$ \\
\hline Recurrent bleeding without ascites & $16(29.6 \%)$ & 19 (33.9\%) \\
\hline \multicolumn{3}{|l|}{ Liver and renal functions (mean \pm SD) } \\
\hline Bilirubin & $1.2 \pm 0.22$ & $1.06 \pm 0.2$ \\
\hline Albumin & $3.27 \pm 0.47$ & $3.18 .1 \pm 0.4$ \\
\hline Serum glutamine transferase & $31 \pm 6.39$ & $32.51 \pm 4.3$ \\
\hline SGPT & $30 \pm 7.37$ & $32.61 \pm 5.1$ \\
\hline Creatinine & $1.1 \pm 0.6$ & $1.01 \pm 0.4$ \\
\hline Urea & $41.2 \pm 9.2$ & $38.16 \pm 6.2$ \\
\hline
\end{tabular}

\section{Encephalopathy in both groups}

Table 3 shows the comparison of both groups regarding the encephalopathy. In both groups, no patients had a history of encephalopathy. After implantation of the stent in the TIPS-LPV group, 8 patients $(14.8 \%, 8 / 54)$ suffered from encephalopathy within the first year, including 3 patients belonging to $\mathrm{HE}$ grade 1,3 belonging to $\mathrm{HE}$ grade 2 , and 2 belonging to $\mathrm{HE}$ grade 3 . They were treated with lactulose ( $10 \mathrm{ml}, 3$ times per day) and ornithine aspartate injection therapy (10 g, intravenous drip, once per day), and they recovered within 1-3 days. In contrast, in the TIPS-RPV group, 19 patients (33.6\%, 19/56) suffered from encephalopathy within the first year follow-up period, including 11 patients belonging to
Table 3 Encephalopathy after TIPS in both studied groups

\begin{tabular}{llll}
\hline Parameter & TIPS-LPV & TIPS-RPV & $P$ value \\
\hline 6-month encephalopathy rate & $5 / 54(9.2)$ & $13 / 56(23.2 \%)$ & $<0.05$ \\
12-month encephalopathy rate & $8 / 54(14.8)$ & $19 / 56(33.6)$ & $<0.05$ \\
Number of encephalopathy attacks & 13 & 33 & $<0.05$ \\
One attack & 8 & 19 & \\
Two attacks & 4 & 12 & \\
Three attacks & 1 & 2 & \\
\hline
\end{tabular}

HE grade 1, 5 belonging to HE grade 2, and 3 belonging to HE grade 3 . They were treated the same as the TIPSLPV and also recovered within 1-4 days. In order to avoid other potential factors influencing $\mathrm{HE}$, we compared the final diameter of the stents in both groups, and there was no significant difference $(P=0.452)$. Regarding the number of encephalopathy attacks within the first year, there were 13 attacks (in 8 patients) for TIPS-LPV compared to 33 attacks (in the 19 patients) for TIPS-RPV $(P<0.05)$.

\section{Re-hospitalizations}

The major causes of re-admission were shunt dysfunction, re-bleeding, encephalopathy, and ascites requiring paracentesis. In the TIPS-LPV, 11 patients were admitted to the hospital at least once during follow-up, and the total number of admissions in this group was 13, as some patients were admitted more than once. Of the 13 readmissions, 5 were for stent dysfunction, 5 were for $\mathrm{HE}, 2$ were for re-bleeding, and 1 was for ascites. In the TIPS-RPV group, 24 patients were admitted to the hospital at least once during follow-up, and the total number of admissions in this group was 39. Of the 39 hospital readmissions, 17 were for stent intervention, 16 were for HE, 5 were for re-bleeding, and 2 were for ascites. The mean number of days of re-hospitalization during the first year surveillance program was $3.7 \pm 1.2$ days in the TIPS-LPV, compared to $5.2 \pm 1.6$ days in the TIPS-RPV $(P<0.05)$.

\section{Discussion}

Reducing portal hypertension with the insertion of TIPS is a well-established interventional procedure. TIPS is used to manage cases of refractory portal hypertension not responding to standard medical treatment such as

Table 2 Follow up 6 month after TIPS in both studied groups

\begin{tabular}{llll}
\hline Parameter & TIPS-LPV & TIPS-RPV & P value \\
\hline 6-month primary patency rate & $51 / 54(94.4)$ & $45 / 56(80.3 \%)$ & $<0.01$ \\
12-month patency rate & $49 / 54(90.7)$ & $41 / 56(73.2)$ & $<0.005$ \\
Number of re-interventions & 5 & 23 & $<0.05$ \\
Mean re-hospitalization time (days) & $3.7 \pm 1.2$ & $4.8 \pm 1.4$ & $<0.05$ \\
\hline
\end{tabular}


variceal bleeding and ascites. There is a debate about TIPS role regarding cirrhotic patients with complications. In this study, the indication for TIPS was for the prevention of significant variceal bleeding with or without ascites. Most of the studies reported a high incidence of the TIPS adverse effects including TIPS stenosis and de novo encephalopathy, especially with bare stents. The latter is closely related to the patient's survival [17]. Encephalopathy rates were widely variable among different studies (30-60\%) [18, 19].

The most preferred approach when doing the standard TIPS is to extend the shunt to the RPV (Fig. 7). The lower anatomical site and horizontal course of the right vein allow safer and easier course of the TIPS needle to get inside its lumen. On the other hand, the selection of the left branch of the portal vein was not widely performed [20]. Eventually, some studies reported important results when selecting the LPV as they found that the LPV approach has a lower incidence of stent occlusion, less encephalopathy, and subsequently, less rehospitalizations than the RPV approach suggesting it as a preferable technique when doing TIPS $[19,20]$.

The study of Chen et al. compared the clinical outcome of the TIPS-RPV and TIPS-LPV. They reported no significant difference in stent patency in the TIPS-LPV group compared to the TIPS-RPV group caused by acute thrombosis pseudointimal and subintimal hyperplasia of the hepatic vein after 3 months, 1 year after TIPS placement [19]. In their study, only bare stents were used not covered stents. Thus, they reported that the shunt dysfunction was not affected by anatomically selected branches of the portal vein. We disagree with Chen and his group regarding differential stent dysfunction, although we used bare stents as well. In our study, we found that the TIPS-LPV group had a primary patency rate significantly higher than the TIPS-RPV. We explained the higher patency by two factors [19]. First is a straight course of the shunt when implanted in the left portal compared to the curved course on the right. In many cases of TIPS-RPV, there would be a kink or loop in the stent that is inevitable when implanted on the right. The second factor is the use of a single stent when using the left portal compared to two stents in some cases. This is attributed to the shorter distance between the hepatic veins and LPV compared to RPV. The use of 2 stents necessitates telescoping of one inside the other that creates an internal circumferential edge of the junctional zone. The later predisposes to thrombosis either acute or in a chronic way. One interesting observation is that the primary patency rate of our bare stents in TIPSLPV might be comparable to patency rates that were reported in literature for covered stents [7].

Encephalopathy is a major determining factor with a great concern of clinicians after TIPS [19]. In our study, we found that patients with TIPS-LPV developed less rates of encephalopathy compared to TIPS-RPV that makes it superior to TIPS-RPV. Luo et al. in their study reported the same finding as their TIPS-LPV patients have less incidence of encephalopathy. These results are due to the balance between the reduction of portal hypertension and liver perfusion improvement from the portal vein. Another explanation by $\mathrm{Chu}$ et al. in an animal study, ammonia content of the mesenteric vein of a rabbit was higher than that of the portal vein branch, splenic vein, and vena cava, as well as ammonia was higher in the right branch portal vein than the left branch, but a typical study on humans is not available yet $[18,20]$.

The major causes of re-admission were shunt dysfunction, re-bleeding, encephalopathy, and ascites requiring paracentesis. Chen et al. found in his study that readmissions and cost of the procedure were less in the group with LPV stent compared to RPV. They explained the reduced hospital re-admission by the reduced

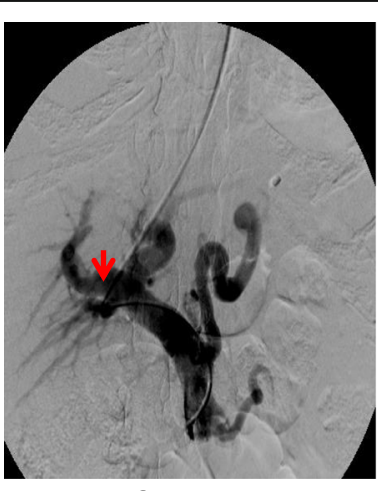

a

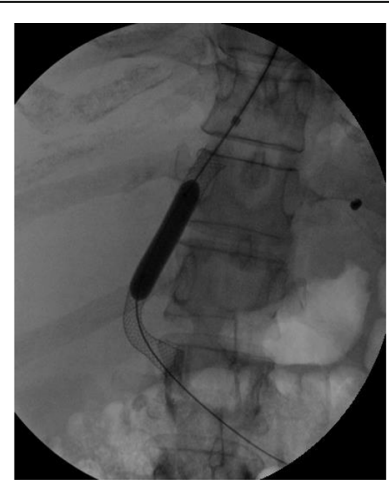

b

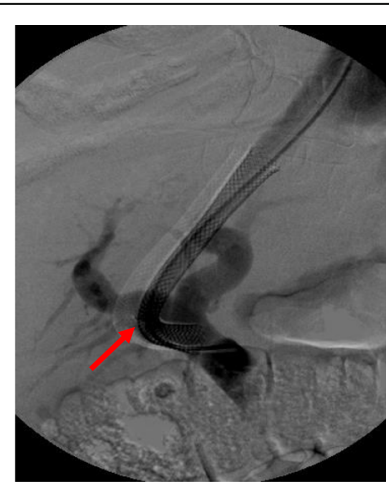

C

Fig. 7 Standard TIPS in a patient of the TIPS-RPV group. The right portal vein (arrowhead) was approached followed by the main portography to measure the pressure (a). Finally, the Wallstent that was delivered shows inevitable angulation (arrow) through the course between the right portal and cava $(\mathbf{b}, \mathbf{c})$ 
incidence of encephalopathy in this group. We confirmed the finding of Chen and his group where patients with TIPS-LPV had lower rates of re-admission compared to those of the TIPS-RPV group. Moreover, the mean hospital stay was reduced in the TIPS-LPV group compared to TIPS-RPV. We explained these two advantages by not only the less frequency of encephalopathy but by the less frequency of shunt dysfunction in the TIPS-LPV as well [19].

Our study has some limitations. The most important limitation is the retrospective design of our study. Of course, this design had the disadvantage that we might include a bias when comparing stent patency and clinical outcome. Another limitation is the heterogeneity of the studied patients where we included patients with BuddChiary. The thrombogenicity of such patients could affect the primary outcome. But considering the matching statistical power for both groups for comparison, we decided to take this bias into account. The third limitation is the non-randomization of the patients. In the future, we need a controlled randomized study to compare both groups.

\section{Conclusion}

TIPS is still a promising technique for the management of portal hypertension. The new TIPS approach is to extend the stent to LPV. This new TIPS approach showed the same clinical efficiency as the standard TIPS-RPV in treating variceal bleeding and ascites. However, it proved a better stent patency with lower rates of encephalopathy and hospital admissions than TIPS through the right branch. We can consider TIPS-LPV as a primary choice when doing TIPS.

\section{Abbreviations}

TIPS: Trans-jugular intra-hepatic porto-systemic shunt; TIPS-LPV: TIPS through left branch portal vein; TIPS-RPV: TIPS through the right portal branch; HE: Hepatic encephalopathy

\section{Acknowledgements}

We acknowledged Dr. Einas Maged, lecturer of community medicine, National Liver Institute, for helping the statistical analysis of the study.

\section{Authors' contributions}

MA put the conception and design of the study, wrote the protocol, and was the radiologist who performed all TIPS cases whether through the right or left portal veins. He contributed in reviewing medical records, collecting and tabulating data, and selecting figures of the study. He also contributed to manuscript writing and approved the draft. HE contributed in the clinical follow-up of the cases at the time of TIPS. He reviewed medical records and the collection of both groups with manuscript drafting and approved the draft. MM contributed to the clinical follow-up of the cases with endoscopic evaluation at the time of TIPS. He reviewed medical records and the collection of both groups with manuscript drafting and approved the draft. AM contributed to the clinical follow-up of the cases at the time of TIPS. He reviewed the medical records and collected data of both groups with manuscript drafting. He also contributed to the clinical follow-up with endoscopic evaluation at the time of TIPS and manuscript drafting and approved the draft. Finally and collectively, all authors have read and approved the manuscript

\section{Funding}

No funding was received for this study.

Availability of data and materials

All data and materials are available when needed.

\section{Ethics approval and consent to participate}

This study was approved by the Research Ethics Committee of the National Liver Institute at Menoufia University in Egypt NLI IRB number 00214/2020 (name of IRB; NLI IRB 00003413). All patients' consent to participate in this research was waived as it is a retrospective study.

\section{Consent for publication}

Being a retrospective study, patients' consent to publish the data contained within this study has been waived by the ethical committee

\section{Competing interests}

The authors or author's institutions have no conflicts of interest which include financial or personal relationships that inappropriately influence their actions.

\section{Author details}

${ }^{1}$ Radiology Department, National Liver Institute, Menoufia University, Menoufia, Shebin Al-Kom, Egypt. ²Department of Diagnostic \& Interventional Imaging, National Liver Institute, Menoufia University, Yassin Abdelghaffar St., Menoufia, Shebin Al-Kom, Egypt. ${ }^{3}$ Hepatology Department, National Liver Institute, Menoufia University, Menoufia, Shebin Al-Kom, Egypt.

Received: 14 July 2020 Accepted: 1 September 2020

Published online: 15 September 2020

\section{References}

1. Merli M, Salerno F, Riggio O et al (1998) Transjugular intrahepatic portosystemic shunt versus endoscopic sclerotherapy for the prevention of variceal bleeding in cirrhosis: a randomized multicenter trial Gruppo Italiano Studio TIPS (G. I. S. T.). Hepatology 27:48-53

2. Ring EJ, Lake JR, Roberts JP et al (1992) Using transjugular intrahepatic portosystemic shunts to control variceal bleeding before liver transplantation. Ann Intern Med 116:304-309

3. Pomier-Layrargues G, Villeneuve JP, Desche^nes M et al (2001) Transjugular intrahepatic portosystemic shunt (TIPS) versus endoscopic variceal ligation in the prevention of variceal rebleeding in patients with cirrhosis: a randomized trial. Gut 48:390-396

4. Salerno F, Camma' C, Enea M, Rossle M, Wong F (2007) Transjugular intrahepatic portosystemic shunt for refractory ascites: a meta-analysis of individual patient data. Gastroenterology 133:825-834

5. Zheng M, Chen Y, Bai J et al (2008) Transjugular intrahepatic portosystemic shunt versus endoscopic therapy in the secondary prophylaxis of variceal rebleeding in cirrhotic patients: meta-analysis update. J Clin Gastroenterol 42:507-516

6. D'Amico G, Luca A, Morabito A, Miraglia R, D'Amico M (2005) Uncovered transjugular intrahepatic portosystemic shunt for refractory ascites: a metaanalysis. Gastroenterology 129:1282-1293

7. Gandini R, Konda D, Simonetti G (2006) Transjugular intrahepatic portosystemic shunt patency and clinical outcome in patients with BuddChiari syndrome: covered versus uncovered stents. Radiology 241:298-305

8. Hassoun Z, Desche^nes M, Lafortune M et al (2001) Relationship between pre-TIPS liver perfusion by the portal vein and the incidence of post-TIPS chronic hepatic encephalopathy. Am J Gast roenterol 96:1205-1209

9. Walser EM, DeLa Pena R, Villanueva-Meyer J, Ozkan O, Soloway R (2000) Hepatic perfusion before and after the transjugular intrahepatic portosystemic shunt procedure: impact on survival. J Vasc Inter $\vee$ Radiol 11: 913-918

10. Luo S-H, Chu J-G, Huang H, Yao K-C (2017) Effect of initial stent position on patency of transjugular intrahepatic portosystemic shunt. World J Gastroenterol 23(26):4779-4787

11. Ferenci P, Lockwood A, Mullen K et al (2002) Hepatic encephalopathydefinition, nomenclature, diagnosis, and quantification: final report of the working party at the 11th World Congresses of Gastroenterology, Vienna, 1998. Hepatology 35:716-721 
12. Zhang $\mathrm{L}$, Huan $\mathrm{H}$, Tong $\mathrm{H}$ et al (2020) Warfarin prevented de novo portal vein thrombosis after transjugular intrahepatic portosystemic shunt A retrospective study. Medicine $99: 2$

13. Stewart CA, Malinchoc M, Kim WR, Kamath PS (2007) Hepatic encephalopathy as a predictor of survival in patients with end-stage liver disease. Liver Transpl 13:1366-1371

14. Salerno F, Merli M, Riggio O et al (2004) Randomized controlled study of TIPS versus paracentesis plus albumin in cirrhosis with severe ascites. Hepatology 40:629-635

15. Li Y-H, Xu Z-Y, Wu H-M, Yang L-H et al (2018) Long-term shunt patency and overall survival of transjugular intrahepatic portosystemic shunt placement using covered stents with bare stents versus covered stents alone. Clin Radiol. 73(6):580-587

16. Kauffmann GW, Richter GM (1999) Transjugular intrahepatic portosystemic stent-shunt (TIPSS): technique and indications. Eur Radiol 9:685-692

17. Colombato $L$ (2007) The role of transjugular intrahepatic portosystemic shunt (TIPS) in the management of portal hypertension. J Clin Gast roenterol 41(Suppl. 3):S344-S351

18. Chu J, Sun X, Piao L et al (2002) Portosystemic shunt via the left branch of portal vein for the prevention of encephalopathy following transjugular intrahepatic portosystemic shunt. Zhong Hua Gan Zang Bing Za Zhi 10: 437-440

19. Chen L, Xiao T, Chen W, Long Q, Li R et al (2009) Outcomes of transjugular intrahepatic shunt through the left branch vs. the right branch of the portal vein in advanced cirrhosis; a randomized trial. Liver International:1101-1109

20. Luo S-H, Chu J-G, He H et al (2019) Targeted puncture of left branch of intrahepatic portal vein in transjugular intrahepatic portosystemic shunt to reduce hepatic encephalopathy. World J Gastroenterol 25(9):1088-1099

\section{Publisher's Note}

Springer Nature remains neutral with regard to jurisdictional claims in published maps and institutional affiliations.

\section{Submit your manuscript to a SpringerOpen ${ }^{\circ}$ journal and benefit from:}

- Convenient online submission

- Rigorous peer review

- Open access: articles freely available online

High visibility within the field

- Retaining the copyright to your article

Submit your next manuscript at $\boldsymbol{\nabla}$ springeropen.com 